\title{
Characterization of Cas proteins for CRISPR-Cas editing in streptomycetes
}

Wan Lin Yeo ${ }^{1 \dagger}$, Elena Heng ${ }^{2 \dagger}$, Lee Ling Tan², Yi Wee Lim, ${ }^{3}$ Yee Hwee Lim³, Shawn Hoon ${ }^{2}$, Huimin

Zhao ${ }^{4}$, Mingzi M. Zhang ${ }^{1,5 *}$, Fong Tian Wong ${ }^{2 *}$

Running title: CRISPR-Cas editing in streptomycetes

1. Metabolic Engineering Research Laboratory, Institute of Chemical and Engineering Sciences, A*STAR,

Singapore

2. Molecular Engineering Laboratory, Biomedical Institutes of Sciences, $A^{*}$ STAR, Singapore

3. Organic Chemistry, Institute of Chemical and Engineering Sciences, A*STAR, Singapore

4. Department of Chemical and Biomolecular engineering, Department of Chemistry, Department of

Biochemistry, University of Illinois at Urbana-Champaign, United States

5. Institute of Molecular and Genomic Medicine, National Health Research Institutes, Taiwan, R.O.C.

"Contributed equally

*Correspondence should be directed to: zhangmz@nhri.org.tw, ftwong@bmsi.a-star.edu.sg

Grants: National Research Foundation, Singapore [NRF2013-THE001-094 to MMZ, FTW, YHL] and the $A^{*} S T A R$ Visiting Investigator Program [HZ]. 


\begin{abstract}
Application of the well-characterized Streptococcus pyogenes CRISPR-Cas9 system in actinomycetes has enabled high efficiency multiplex genome editing and CRISPRi-mediated transcriptional regulation in these prolific bioactive metabolite producers. Nonetheless, SpCas9 has its limitations and can be ineffective depending on the strains and target sites. Here, we built and tested alternative CRISPR-Cas constructs based on the standalone pCRISPomyces-2 editing plasmid. We showed that Streptococcus thermophilus CRISPR1 (sth1Cas9), Staphylococcus aureus Cas9 (saCas9), and Francisella tularensis subsp. Novicida U112 Cpf1 (fnCpf1) are functional in multiple streptomycetes enabling efficient homology directed repair (HDR)-mediated knock-in and deletion. In strains where spCas9 was nonfunctional, these alternative Cas systems enabled precise genomic modifications within biosynthetic gene clusters for the discovery, production and diversification of natural products. These additional Cas proteins provide us with the versatility to overcome the limitations of individual CRISPR-Cas systems for genome editing and transcriptional regulation of these industrially important bacteria.
\end{abstract}

Keywords: CRISPR-Cas, Streptomyces, genome editing 


\section{Introduction}

The CRISPR-Cas technology has revolutionized biological research and the biotech industry. Since the first application of Class 2 CRISPR-Cas systems to edit genomes (Cong, et al, 2013), the number and diversity of CRISPR-Cas applications (Adli, 2018) have increased exponentially. The rapid adoption of this technology is due to its capability for precise editing and ease of use - a short RNA transcript is sufficient to guide the nuclease to its desired target, as well as the wide range of organisms that it can be applied in. These advantages have led us and other labs to explore CRISPR-Cas editing in streptomycetes (Li, et al, 2018, Zhang, et al, 2018, Tao, et al, 2018), which are prolific producers of bioactive secondary metabolites. Previously, we demonstrated that CRISPR-Cas increases the speed and throughput of genome editing compared to the traditional two step selection-counterselection protocol, allowing rapid genetic access to natural products encoded in the genomes of hitherto genetically intractable streptomycetes (Zhang, et al, 2018).

To date, Streptomyces strains have been mostly engineered using the well-characterized Streptococcus pyogenes Cas9 (spCas9, Tao, et al, 2018). Yet as we have observed in our labs and others (Moreb, et al, 2017, Cui, et al, 2018), a major bottleneck is that many bacteria strains still cannot be edited by S. pyogenes Cas9. In some examples, after a successful introduction of recombinant CRISPR-Cas systems, some bacteria can still evade modifications induced by CRISPR-Cas9 double stranded breaks with RecA-dependent repair (Moreb, et al, 2017). In other strains or even in certain target sites of previously editable strains, no exconjugants can be obtained in the presence of Cas 9 and its guide RNA, possibly due to off-target nuclease-induced toxicity or tight binding of Cas9 to nontargets in the absence of guide RNAs (Cui, et al, 2018). To overcome similar bottlenecks in streptomycetes, we were motivated to expand the CRISPR-Cas toolbox. In this study, we compared different Class 2 Cas proteins and showed that these alternative Cas proteins allow us to overcome the bottlenecks faced with using spCas9 to facilitate natural product discovery in these industrially important bacteria. 


\section{Materials and methods}

\section{Reagents and Media}

Unless otherwise indicated, all reagents are obtained from Sigma. $1 \mathrm{~L}$ of MGY medium contains $10 \mathrm{~g}$ malt extract broth, $4 \mathrm{~g}$ Bacto yeast extract (BD Biosciences), $4 \mathrm{~g}$ glucose (1st Base, Axil Scientific) and for MGY agar plates, an additional $20 \mathrm{~g}$ of Bacto agar (BD Biosciences). ISP Medium No. 4 agar (BD Biosciences) for Streptomyces sp. NRRL S-244. Conjugation experiments involving WM6026 and WM3780 E. coli strains were performed on R2 agar without sucrose: $0.25 \mathrm{~g} \mathrm{~K}_{2} \mathrm{SO}_{4}, 10.12 \mathrm{~g}$ $\mathrm{MgCl}_{2} \bullet 6 \mathrm{H}_{2} \mathrm{O}, 10 \mathrm{~g}$ glucose, $0.1 \mathrm{~g}$ Bacto casamino acids (BD Biosciences), $5.73 \mathrm{~g}$ TES, $20 \mathrm{~g}$ agar in 1

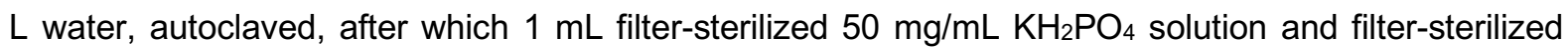
$2.94 \mathrm{~g} \mathrm{CaCl}_{2} \cdot 2 \mathrm{H}_{2} \mathrm{O}$ and $3 \mathrm{~g} \mathrm{~L}$-proline in $5 \mathrm{~mL} 1 \mathrm{~N} \mathrm{NaOH}$ were added to the medium.

\section{Strains and Growth conditions}

Unless otherwise indicated, strains are propagated in MGY medium at $30^{\circ} \mathrm{C}$. Spore preparations and conjugation protocols were similar as described before (Aínsa, et al, 2000). For spore preparations, 1:1000 of a spore preparation or 1:100 dilution of a saturated seed culture is plated on MGY or ISP Medium No. 4 plates and incubated at $30^{\circ} \mathrm{C}$ until thick spores are observed. Spores were removed from the plate using $5 \mathrm{~mm}$ glass beads (Sigma) and resuspended in sterile TX buffer (50 mM Tris $\mathrm{pH}$ 7.4, $0.001 \%(\mathrm{v} / \mathrm{v})$ Triton $\mathrm{X}$ ) by vigorous vortexing for $2 \mathrm{~min}$. The suspension was then passed through a syringe containing a cotton ball. The eluant containing free spores were pelleted by spinning at maximum speed in an Eppendorf 5810R centrifuge for $10 \mathrm{~min}$, resuspended in $1 \mathrm{~mL}$ sterile water and pelleted again. The spores were then resuspended in water and stored at $-80^{\circ} \mathrm{C}$. A typical spore prep contains $\sim 10^{6}-10^{7}$ spores $/ \mathrm{mL}$ as determined by serial dilution plating.

\section{Target site prediction}

Target sites are predicted using modifications to PhytoCRISP-Ex software (Rastogi, et al, 2016). Potential sites are given as sites where seed regions (last 15 bases including PAM sequences) do not have $100 \%$ match with off-targets.

\section{Construction of genome editing plasmids}

The different Cas proteins were codon-optimized based on the codon usage of Streptomyces coelicolor and synthesized by Genscript. All DNA manipulations were carried out in OmniMAX ${ }^{\mathrm{TM}}$ (Thermo Fisher). 
Primers used in this study are listed in Supplementary Table 1. Restriction enzymes were obtained from New England Biolabs. Protospacers were first inserted via Bbsl-mediated Golden Gate Assembly before introduction of the respective homology flanks via Gibson assembly, as previously described (Zhang, et al, 2017).

\section{Interspecies conjugation}

Promoter knock-in constructs were used to transform conjugating E. coli strains and colonies with apramycin resistance $(50 \mathrm{mg} / \mathrm{L})$ were picked into LB with apramycin. WM6026 requires diaminopimelic acid $(0.3 \mathrm{mM})$ in LB for growth and it was added to LB for subsequent wash and resuspension steps. Overnight cultures were diluted 1:100 into fresh LB with antibiotics and grown to an $\mathrm{OD}_{600}$ of $0.4-0.6$. $400 \mu \mathrm{L}$ of the culture was pelleted, washed twice and resuspended in LB without antibiotics. The washed $E$. coli cells were then mixed with spores at 1:5 volume ratio and spotted on $\mathrm{R} 2$ without sucrose plates. After incubation for $16-20 \mathrm{~h}$ at $30^{\circ} \mathrm{C}$, the plates were flooded with nalidixic acid and apramycin and incubated until exconjugants appear. Exconjugants were streaked onto MGY or ISP Medium No. 4 plates containing apramycin at $30^{\circ} \mathrm{C}$ followed by restreaking to MGY or ISP Medium No. 4 plates at $37{ }^{\circ} \mathrm{C}$ to cure the CRISPR-Cas9 plasmid containing a temperature-sensitive origin of replication. Apramycin-sensitive clones growing at $37^{\circ} \mathrm{C}$ were then subjected to validation of promoter knock-in and genome editing as described below.

\section{Validation of promoter knock-in and genome editing}

Genomic DNA from wild type and exconjugants from the indicated strains were isolated from liquid cultures using the Blood and Tissue DNeasy kit (Qiagen) after pretreating the cells with $20 \mathrm{mg} / \mathrm{mL}$ lysozyme for $0.5-1 \mathrm{~h}$ at $30^{\circ} \mathrm{C}$. PCR was performed using control primers beyond the homology regions (Supplementary Table 1) with KODXtreme Taq polymerase (Millipore). Where indicated, PCR products were subjected to digest with specific restriction enzymes to differentiate between PCR products of wild type genomic sequences and successful genome editing by knock-ins. Positive samples were purified using ExoSAP-IT ${ }^{\mathrm{TM}}$ (Affymetrix USB) and validated by Sanger sequencing (Figure S1, S2). 


\section{Fermentation, extraction and NMR of phosphonate compound}

Liquid seed cultures (2 mL MGY) of wild type and engineered S. sp. NRRL S-244 strains were inoculated from a plate or spore stock in $14 \mathrm{~mL}$ culture tubes. Seed cultures were incubated at $30^{\circ} \mathrm{C}$ with 250 rpm shaking until achieving turbidity or high particle density (2 days). Seed cultures were diluted 1:100 into $50 \mathrm{~mL}$ of MGY broth in $250 \mathrm{~mL}$ baffled flasks containing $\sim 30-405 \mathrm{~mm}$ glass beads and incubated at $30{ }^{\circ} \mathrm{C}$ with $250 \mathrm{rpm}$ shaking for 7 days. The cultures were harvested by pelleting at maximum speed in an Eppendorf 5810R centrifuge for $10 \mathrm{~min}$. The supernatants were split into two 50 $\mathrm{mL}$ falcon tubes. Culture supernatants were lyophilized and then extracted with equal volume of methanol, before evaporated to dryness.

The extracts were dissolved in $\mathrm{D}_{2} \mathrm{O}$ and transferred to a $5 \mathrm{~mm}$ NMR tube for NMR analysis. ${ }^{31} \mathrm{P}-\mathrm{NMR}$ has been acquired using a Bruker DRX-400 spectrometer. Proton decoupled ${ }^{31} \mathrm{P}-\mathrm{NMR}$ spectra are referenced to an external $\mathrm{H}_{3} \mathrm{PO}_{4}(\mathrm{aq})$ standard $(\delta 0.0 \mathrm{ppm})$. All samples have been acquired for 2000 scans. Comparison of production titer of various strains was done by spiking in known amounts of authentic FR-900098 (Sigma) as reference standard. 


\section{Results}

\section{Alternative Cas proteins for genome editing of streptomycetes}

In this study, we tested three Cas proteins in addition to our original spCas9 construct for genome editing in streptomycetes (Figure 1). The Cas proteins include Type II Cas from S. pyogenes (spCas9), Streptococcus thermophilus CRISPR1 (sth1Cas9, Horvath, et al, 2008), Staphylococcus aureus (saCas9, Ran, et al, 2015) and Francisella tularensis subsp. Novicida U112's Type V-A Cas (fnCpf1, Zetsche, et al, 2015). Sth1Cas9 and saCas9 are Type II Cas proteins with similar editing efficiencies but are about $25 \%$ smaller in size compared to spCas9 (Horvath, et al, 2008; Ran, et al, 2015). fnCpf1 is a Type V-A single-effector nuclease that is closer in size to spCas9 (Zetsche, et al, 2015). Both Class 2 CRISPR-Cas systems utilize RNA guides to target double stranded DNA with several key differences (Zetsche, et al, 2017, Figure 1). Type II results in a blunt cut whilst Type V-A yields a staggered cut that is preferential towards homology directed repair (HDR) instead of the error-prone non-homologous end-joining (NHEJ) (Figure 1A). Unlike Type II Cas proteins, Type V-A proteins do not require a tracrRNA and a separate RNA processing protein since pre-crRNA can be wholly processed by the nuclease effector. This difference in crRNA processing for Type V-A class proteins allow multiple targets to be transcribed on a single transcript for more rapid and facile multiplexing (Cobb, et al, 2014). The protospacer adjacent motifs (PAMs) for Type II proteins are also more GC-rich compared to the AT-rich PAMs for Type V-A (Figure 1B). Due to GC-rich streptomycete genomes, spCas9 provides the highest number of targets compared to the other Cas proteins (Table 1), but have proven ineffective in certain contexts such as those described later in this study. In general, we expect the use of different Cas proteins with distinct PAM requirements to increase our target density, which would be advantageous when targeting short intergenic regions of the genome.

To test the utility of these different Cas proteins in different Streptomyces strains, we chose to focus on the activation and engineering of biosynthetic gene clusters (BGCs), which involve the insertion of small promoter elements into short intergenic regions and genetic manipulation of single genes. The Cas constructs were based on the all-in-one pCRISPomyces-2 editing plasmid (Addgene \#61737, Cobb, et al, 2014) with the respective Cas protein and sgRNA/crRNA under the control of the two distinct promoters (Figure 1C). The final editing constructs was constructed by Golden Gate assembly to insert the targeting guide sequences, followed by the addition of homology repair templates at the Xbal site 
using isothermal assembly as previously described (Zhang, et al, 2017). We sought to target the different Cas proteins to the same genomic region as much as possible, but this is not always possible due to differences in PAM sequence requirements. Choices of target sites and homology repair templates were further limited by the short sequence targeted and our decision to minimize disruption to native BGC sequences. While differences in repair templates are mostly minimal and not expected to have significant impact on editing efficiencies, we acknowledge that selection of target sites is important for editing efficiency and should be considered when comparing the different Cas proteins.

\section{Cas proteins are functional in Streptomyces albus}

We first validated the functionality of the different Cas proteins for genome editing in Streptomyces albus, a model streptomycete with high conjugation efficiencies. Target sites for all four Cas proteins can be found in the 280 bp target intergenic region of the indigoidine cluster (Figure 2). Consistent with our previous study (Zhang, et al, 2017), knock-in efficiency of the kasO* promoter was increased when spCas9 was targeted by the sgRNA to the region upstream of gene encoding for indigoidine synthase compared to the control where no guide was provided (100\% vs $37 \%)$. The other three Cas proteins also yielded high editing efficiencies of $87-100 \%$ as validated by sanger sequencing (Table 2). These results demonstrate that the different Cas proteins are functional in $S$. albus and may be used in place of spCas 9 for genome editing of Streptomyces strains.

\section{Alternative Cas proteins enable genome editing in previously inaccessible contexts}

After validating that the codon-optimized Cas proteins are functional and can be used in place of spCas9 to increase promoter knock-in efficiency in $S$. albus, we next examined how these Cas proteins will perform in applications where spCas9 has failed. Previous attempts using spCas9 to introduce a strong constitutive kasO* promoter in front of a putative Streptomyces antibiotic regulatory protein (SARP) to increase production of a low-yielding phosphonate compound in Streptomyces sp. NRRL S-244 had failed with no observed exconjugants (Figure 3A, Table 2). Highlighting the need for multiple Cas proteins, we were unable to find target sequences for sth1Cas9 (PAM NNAGAA) within range of the 64 bp target intergenic region. Nonetheless with saCas9, we managed to obtain a small number of exconjugants were observed with $100 \%$ editing efficiency. With fnCpf1, more exconjugants were observed with $100 \%$ editing efficiency (Table 2). In the end, a 10-fold upregulation in phosphonate 
production was also observed and validated in shake flask fermentation of two edited strains (Figure 3C).

In another example, spCas9 has worked robustly in Streptomyces roseosporus for both insertions and deletions for multiple BGCs (Zhang, et al, 2017, Lim, et al, 2018), but has failed in a specific context. Attempts to delete a specific glycosyltransferase aurS5 gene in a polyene macrolactam BGC (Lim, et al, 2018) yielded no exconjugants in the presence of spCas9 and a variety of guide RNAs targeting different locations (Figure 4). Interestingly, in the absence of a functional guide, edits could be observed, possibly a result of innate homologous recombination in the presence of the editing template (Table 2). In this case, alternative Cas proteins circumvented this problem with $100 \%$ editing efficiency observed for two saCas9 targets (Figure 4, Table 2).

\section{Discussion}

Here, we present alternative CRISPR-Cas systems for genome editing in streptomycetes and showcase their potential to circumvent some of the limitations associated with the widely used and well characterized S. pyogenes CRISPR-Cas system (Zhang, et al, 2018, Tao, et al, 2018). While Cpf1 systems have recently examined for streptomycetes ( $\mathrm{Li}$, et al, 2018), this would be the first application of sth1Cas9 and saCas9 systems in streptomycetes. While the different Cas proteins all work in the model streptomycete $S$. albus, the alternative Cas toolbox proved to be useful in achieving precise genomic modifications within biosynthetic gene clusters for the discovery, production and diversification of natural products in contexts where spCas9 has failed. We also expect this expanded Cas toolbox to be relevant to the engineering of rare actinomycetes (Liu, et al, 2018). With these observations, this study also provides the motivation for systematic evaluation of different CRISPR-Cas systems in the genetic editing of Streptomyces strains. Limited by short target sequences, we did not compare relative efficiencies for the different systems, which would require a large scale study involving multiple targets for each Cas protein. For example, it remains to be determined if Type V-A Cpf1 provides an advantage in promoting HDR in streptomycetes (Jiang, et al, 2017) compared to Type II Cas proteins since fnCpf1 and saCas 9 can achieve the same editing efficiency in the cases that we have tested. 
The collection of CRISPR-Cas systems that have been characterized and harnessed for various applications has rapidly expanded (Cong, et al, 2013; Zetsche, et al, 2017), but it remains a challenge to edit the genomes of most bacteria due to the lack of robust HDR and NHEJ. In E. coli, this is circumvented by introducing additional phage recombineering machinery (Ronda, et al, 2016; Reisch, et al, 2015), which may not be applicable in other bacteria. In organisms where the spCas9 system has failed due to low efficiency or high toxicity, the use of alternative CRISPR-Cas systems has been successful (Adli, 2018; Rock, et al, 2017; Sun, et al, 2018). Therefore, until we can better understand the mechanisms and rules for successful precise editing by different CRISPR-Cas systems (Chakrabarti, et al, 2018), having a diverse selection of functional Cas proteins provides us with the versatility to overcome the limitations of individual systems. Beyond genome editing, multiplexing with orthogonal catalytically inactive Cas proteins with different guide specificities may allow transcriptional control of different gene subsets in actinomycetes using CRISPR interference (Rock, et al, 2017; Tong, et al, 2015).

\section{Acknowledgments}

This work is supported by the Agency for Science, Technology and Research (A*STAR), Singapore and National Research Foundation, Singapore [NRF2013-THE001-094 to MMZ, FTW, YHL] and the A*STAR Visiting Investigator Program [HZ]. The authors declare no conflict of interest.

\section{References}

Adli, M. (2018). The CRISPR tool kit for genome editing and beyond. Nature communications, 9(1), 1911._doi: 10.1038/s41467-018-04252-2.

Aínsa, J. A. (2000). Practical Streptomyces Genetics. T. Kieser, MJ Bibb, MJ Buttner, KF Chater, DA Hopwood. International Microbiology, 3(4), 260-261.

Chakrabarti, A. M., Henser-Brownhill, T., Monserrat, J., Poetsch, A. R., Luscombe, N. M., \& Scaffidi, P. (2018). Target-specific precision of CRISPR-mediated genome editing. Molecular cell. doi: 10.1016/j.molcel.2018.11.031. 
Cobb, R. E., Wang, Y., \& Zhao, H. (2014). High-efficiency multiplex genome editing of Streptomyces species using an engineered CRISPR/Cas system. ACS synthetic biology, 4(6), 723-728. doi: 10.1021/sb500351f.

Cong, L., Ran, F. A., Cox, D., Lin, S., Barretto, R., Habib, N., ... \& Zhang, F. (2013). Multiplex genome engineering using CRISPR/Cas systems. Science, 1231143. doi:

10.1126/science.1231143.

Cui, L., Vigouroux, A., Rousset, F., Varet, H., Khanna, V., \& Bikard, D. (2018). A CRISPRi screen in E. coli reveals sequence-specific toxicity of dCas9. Nature communications, 9(1), 1912. Doi: 10.1038/s41467-018-04209-5.

Horvath, Philippe, et al. "Diversity, activity, and evolution of CRISPR loci in Streptococcus thermophilus." Journal of bacteriology 190.4 (2008): 1401-1412. doi: 10.1128/JB.01415-07. Jiang, Y., Qian, F., Yang, J., Liu, Y., Dong, F., Xu, C., ... \& Wang, R. (2017). CRISPR-Cpf1 assisted genome editing of Corynebacterium glutamicum. Nature communications, 8, 15179. doi: 10.1038/ncomms15179.

Li, L., Wei, K., Zheng, G., Liu, X., Chen, S., Jiang, W., \& Lu, Y. (2018). CRISPR-Cpf1-assisted multiplex genome editing and transcriptional repression in Streptomyces. Appl. Environ. Microbiol., 84(18), e00827-18. doi: 10.1128/AEM.00827-18.

Lim, Y. H., Wong, F. T., Yeo, W. L., Ching, K. C., Lim, Y. W., ... \& Zhao, H., (2018). Auroramycin, a potent antibiotic from Streptomyces roseosporus by CRISPR-Cas9 activation. ChemBioChem. doi: 10.1002/cbic.201800266.

Liu, Y., Wei, W. P., \& Ye, B. C. (2018). High GC Content Cas9-Mediated Genome-Editing and Biosynthetic Gene Cluster Activation in Saccharopolyspora erythraea. ACS synthetic biology, 7(5), 1338-1348. doi: 10.1021/acssynbio.7b00448. 
Moreb, E. A., Hoover, B., Yaseen, A., Valyasevi, N., Roecker, Z., Menacho-Melgar, R., \& Lynch,

M. D. (2017). Managing the SOS Response for Enhanced CRISPR-Cas-Based Recombineering in E. coli through Transient Inhibition of Host RecA Activity. ACS synthetic biology, 6(12), 22092218. doi: $10.1021 / a c s s y n b i o .7 b 00174$.

Ran, F. A., Cong, L., Yan, W. X., Scott, D. A., Gootenberg, J. S., Kriz, A. J., ... \& Koonin, E. V. (2015). In vivo genome editing using Staphylococcus aureus Cas9. Nature, 520(7546), 186. doi: 10.1038/nature14299.

Rastogi, A., Murik, O., Bowler, C., \& Tirichine, L. (2016). PhytoCRISP-Ex: a web-based and stand-alone application to find specific target sequences for CRISPR/CAS editing. BMC bioinformatics, 17(1), 261. doi: 10.1186/s12859-016-1143-1

Reisch, C. R., \& Prather, K. L. (2015). The no-SCAR (Scarless Cas9 Assisted Recombineering) system for genome editing in Escherichia coli. Scientific reports, 5, 15096. doi: 10.1038/srep15096.

Rock, J. M., Hopkins, F. F., Chavez, A., Diallo, M., Chase, M. R., Gerrick, E. R., ... \& Schnappinger, D. (2017). Programmable transcriptional repression in mycobacteria using an orthogonal CRISPR interference platform. Nature microbiology, 2(4), 16274. doi: 10.1038/nmicrobiol.2016.274.

Ronda, C., Pedersen, L. E., Sommer, M. O., \& Nielsen, A. T. (2016). CRMAGE: CRISPR optimized mage recombineering. Scientific reports, 6, 19452. doi: 10.1038/srep19452.

Sun, B., Yang, J., Yang, S., Ye, R. D., Chen, D., \& Jiang, Y. (2018). A CRISPR-Cpf1-Assisted Non-Homologous End Joining Genome Editing System of Mycobacterium smegmatis. Biotechnology journal, 13(9), 1700588. doi: 10.1002/biot.201700588. 
Tao, W., Yang, A., Deng, Z., \& Sun, Y. (2018). CRISPR/Cas9-based editing of Streptomyces for discovery, characterization and production of natural products. Frontiers in microbiology, 9, 1660. doi: 10.3389/fmicb.2018.01660.

Tong, Y., Charusanti, P., Zhang, L., Weber, T., \& Lee, S. Y. (2015). CRISPR-Cas9 based engineering of actinomycetal genomes. ACS synthetic biology, 4(9), 1020-1029. doi: 10.1021/acssynbio.5b00038.

Weber, T., Blin, K., Duddela, S., Krug, D., Kim, H. U., Bruccoleri, R., ... \& Breitling, R. (2015). antiSMASH 3.0-a comprehensive resource for the genome mining of biosynthetic gene clusters. Nucleic acids research, 43(W1), W237-W243. doi: 10.1093/nar/gkv437.

Zetsche, B., Gootenberg, J. S., Abudayyeh, O. O., Slaymaker, I. M., Makarova, K. S., Essletzbichler, P., ... \& Koonin, E. V. (2015). Cpf1 is a single RNA-guided endonuclease of a class 2 CRISPR-Cas system. Cell, 163(3), 759-771. doi: 10.1016/j.cell.2015.09.038.

Zetsche, B., Heidenreich, M., Mohanraju, P., Fedorova, I., Kneppers, J., DeGennaro, E. M., ... \& Wu, W. Y. (2017). Multiplex gene editing by CRISPR-Cpf1 using a single crRNA array. Nature biotechnology, 35(1), 31. doi: 10.1038/nbt.3737.

Zhang, M. M., Wong, F. T., Wang, Y., Luo, S., Lim, Y. H., Heng, E., ... \& Zhao, H. (2017). CRISPR-Cas9 strategy for activation of silent Streptomyces biosynthetic gene clusters. Nature chemical biology, 13(6), 607. doi: 10.1038/nchembio.2341. 
Table 1. In silico analyses of potential target sites for the indicated Cas proteins and their respective preferred PAMs. Length of target sequence for each case is indicated in brackets. The most preferred PAM sequences for the respective Cas9 proteins were used for this analysis.

\begin{tabular}{cccc}
\hline & \multicolumn{3}{c}{ Potential target sites } \\
\cline { 2 - 4 } Cas/PAM & $\begin{array}{c}\text { Streptomyces albus } \\
\text { J1074 (3.8 kb) }\end{array}$ & $\begin{array}{c}\text { Streptomyces sp. } \\
\text { NRRL S-244 (2.0 kb) }\end{array}$ & $\begin{array}{c}\text { Streptomyces roseosporus } \\
\text { NRRL 15998 (5.7 kb) }\end{array}$ \\
\hline spCas/NGG & 228 & 109 & 327 \\
saCas9/NNGRRT & 54 & 32 & 85 \\
sth1Cas9/NNAGAA & 9 & 3 & 19 \\
fnCpf1/TTN & 63 & 29 & 117 \\
\hline
\end{tabular}


Table 2. Editing efficiencies and exconjugant output from Cas experiments.

\begin{tabular}{|c|c|c|}
\hline Cas constructs & Editing efficiency ${ }^{\dagger}$ & \# exconjugants ${ }^{\ddagger}$ \\
\hline \multicolumn{3}{|c|}{ Streptomyces albus J1074 } \\
\hline $\begin{array}{l}\text { No protospacer control (spCas9, } \\
\text { template } 1)\end{array}$ & $37 \%(3 / 8)$ & $>400$ \\
\hline spCas9 (template 1) & $100 \%(8 / 8)$ & $>400$ \\
\hline saCas9 (template 1) & $87 \%(7 / 8)$ & $>400$ \\
\hline sth1Cas9 (template 2) & $100 \%(8 / 8)$ & $>400$ \\
\hline fnCpf1 (template 2) & $87 \%(7 / 8)$ & $>400$ \\
\hline \multicolumn{3}{|c|}{ Streptomyces sp. NRRL S-244 } \\
\hline No protospacer control (spCas9) & $0 \%(0 / 8)$ & 29 \\
\hline spCas9 & No exconjugants & 0 \\
\hline saCas9 & $100 \%(3 / 3)$ & 3 \\
\hline fnCpf1 & $100 \%(8 / 8)$ & 38 \\
\hline \multicolumn{3}{|c|}{ Streptomyces roseosporus NRRL 15998} \\
\hline No protospacer control (spCas9) & $12.5 \%(1 / 8)$ & 89 \\
\hline spCas9 (targets 1-3) $\S$ & No exconjugants & 0 \\
\hline saCas9 (target 1) & $100 \%(3 / 3)$ & 3 \\
\hline saCas9 (target 2) & $100 \%(2 / 2)$ & 2 \\
\hline
\end{tabular}

† Editing efficiency given by the number of correct clones validated by sanger sequencing over the total number of clones picked.

$¥$ Number of exconjugants observed per $100 \mu \mathrm{L}$ of spore preparation used in each conjugation. A typical spore prep contains $\sim 10^{6}-10^{7}$ spores/ $\mathrm{mL}$ as determined by serial dilution plating.

$\S$ In multiple independent experiments with spCas9 targeting three different sites, no exconjugants were observed. 
A

Cas complex and target binding

Homology directed repair
Type II Cas9
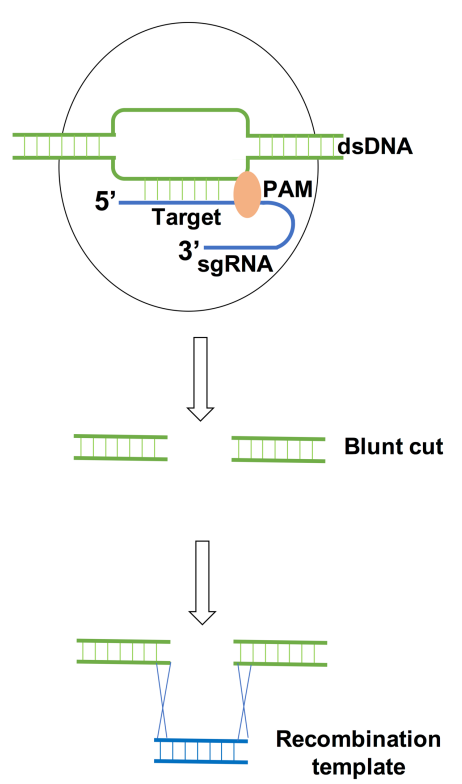

Precise edited DNA

B

CRISPR (Protein, size)

Streptococcus pyogenes spCas $9,4.1 \mathrm{~kb}$

Streptococcus thermopilus 1 sth1Cas9, $3.4 \mathrm{~kb}$

Staphylococcus aureus saCas9, $3.2 \mathrm{~kb}$

Francisella novicida fnCpf1, $3.9 \mathrm{~kb}$

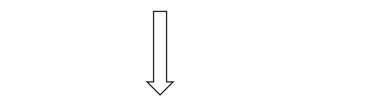

PAM sequence
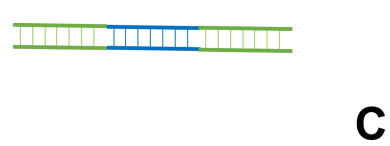

\section{C}

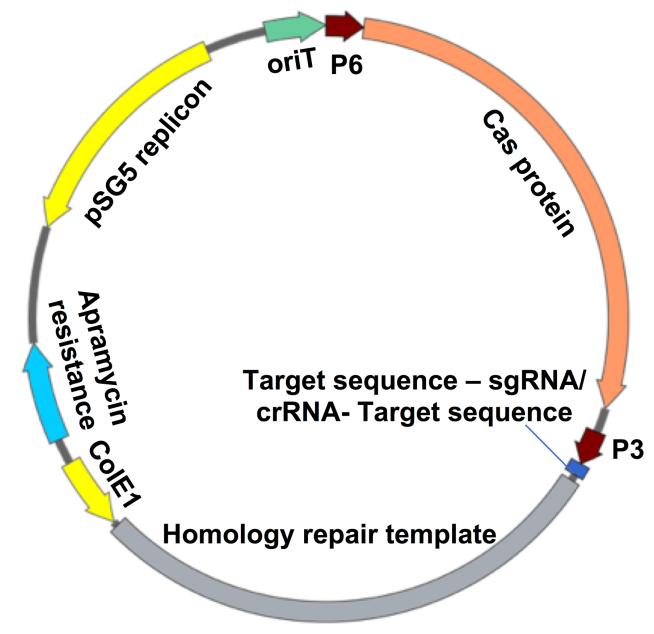

Type V-A Cpf1

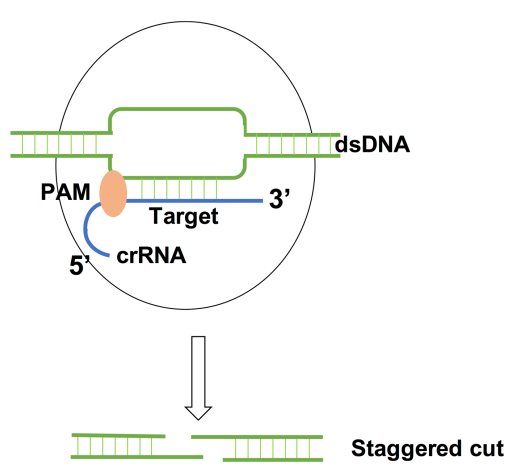

Figure 1. CRISPR-Cas systems used in this study. (A) Comparison of Type II and Type V-A Class

2 Cas systems. (B) Comparison of different Cas proteins and their target sequence requirements. (C) General map of all-in-one editing CRISPR-Cas constructs for one-step genome editing of streptomycetes. PAM, protospacer adjacent motif. P3 and P6 are promoters. 


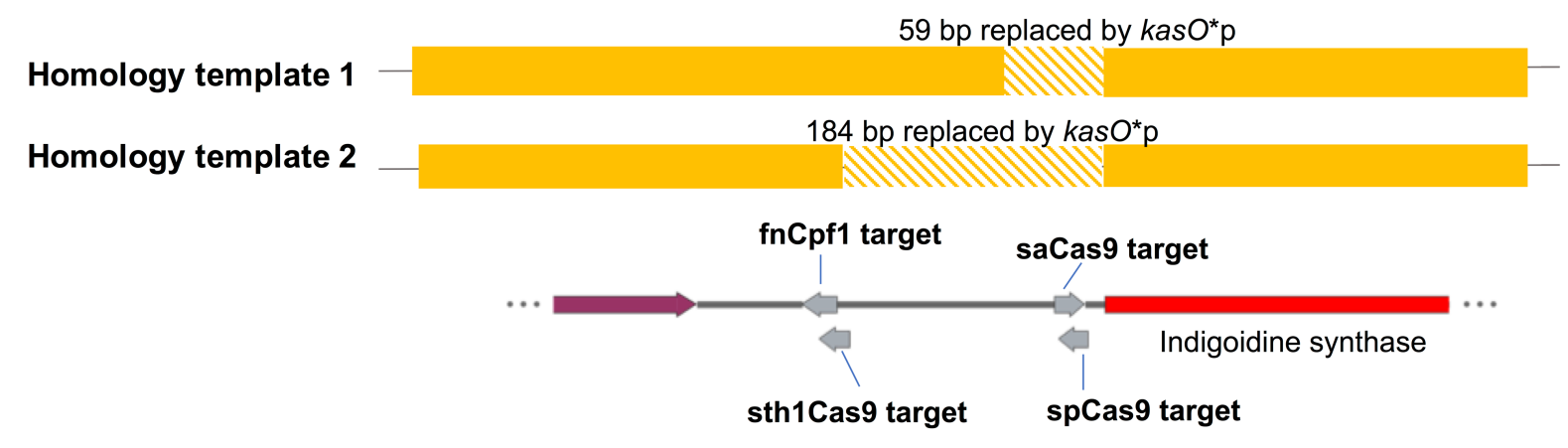

Figure 2. Target sequences for the different Cas proteins in $S$. albus for insertion of kas $O^{*} \mathrm{p}$ in

front of indigoidine synthase. Intergenic region between the two genes are $280 \mathrm{bp}$. Homology arms of the editing templates (solid yellow) are aligned to the scheme of the genome and are designed to minimize disruption to native genome. For sth1Cas9, since NNAGAA is not available with the target sequence, the protospacer for sth1Cas9 was designed using an alternative NNGGAA PAM (Jiang, et al, 2017). 


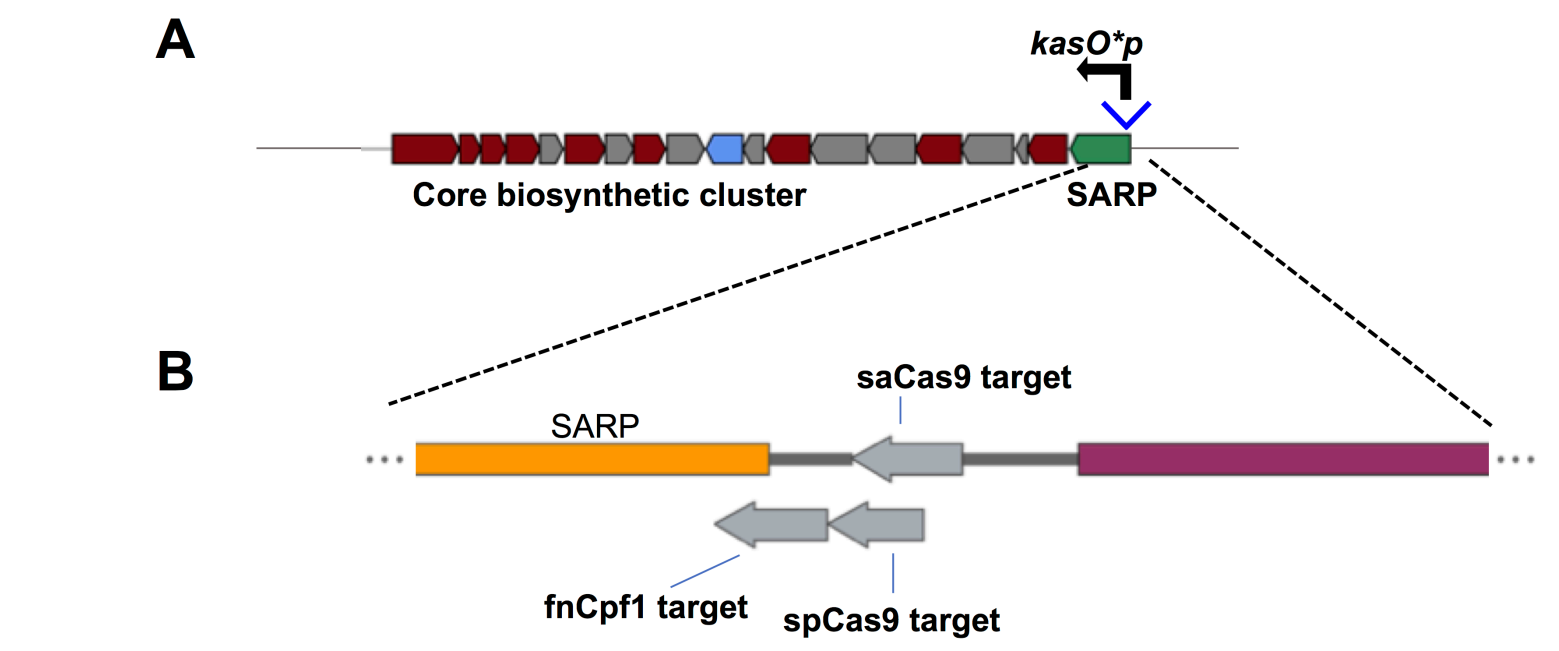

\section{Homology template}

64 bp replaced by kasO*p

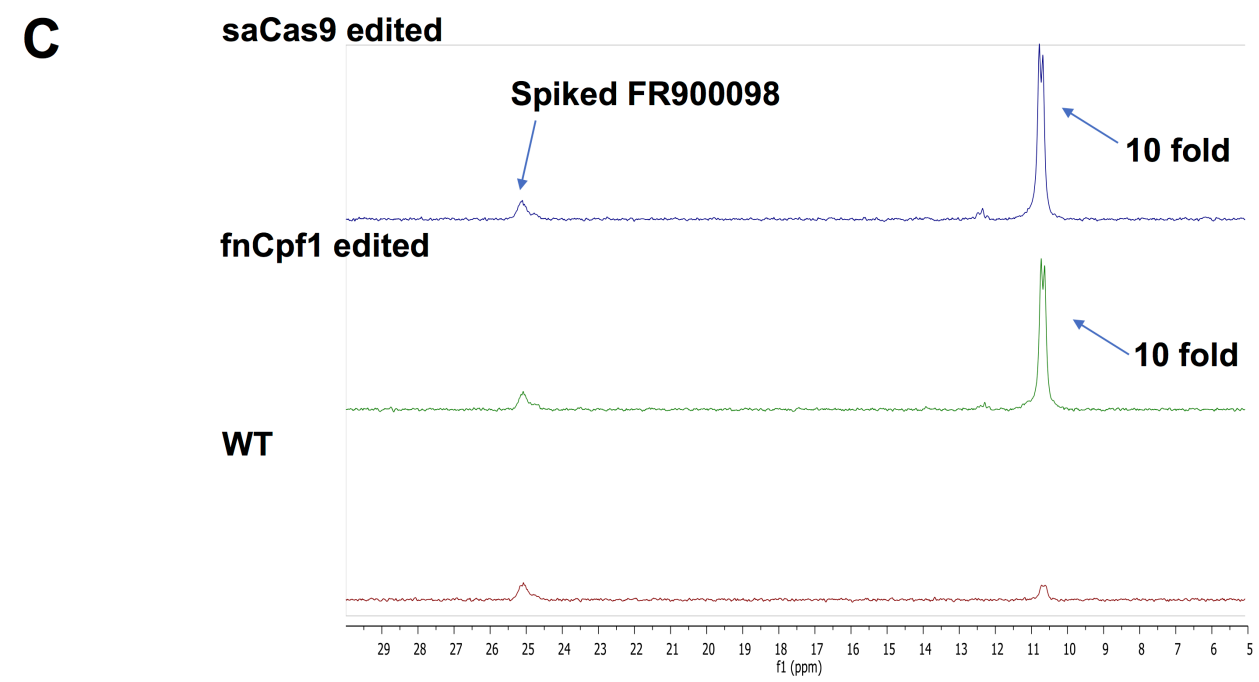

Figure 3. Upregulation of phosphonate production in Streptomyces sp. NRRL S-244. (A) Introduction of $k a O^{*} \mathrm{p}$ in front of SARP within the putative phosphonate BGC as annotated by AntiSMASH (Weber, et al, 2015). Red encodes biosynthetic enzymes, blue for transport protein and green for regulatory proteins. (B) Positions of target sequences for indicated Cas proteins. Homology arms of the editing templates (solid yellow) are aligned to the scheme of the genome. (C) ${ }^{31} \mathrm{P}-\mathrm{NMR}$ for two edited clones in comparison to wild type strain. Spiked FR-900098 is used as an internal standard for fold change calculations. 
bioRxiv preprint doi: https://doi.org/10.1101/526996; this version posted January 22, 2019. The copyright holder for this preprint (which was not certified by peer review) is the author/funder, who has granted bioRxiv a license to display the preprint in perpetuity. It is made available under aCC-BY-NC-ND 4.0 International license.

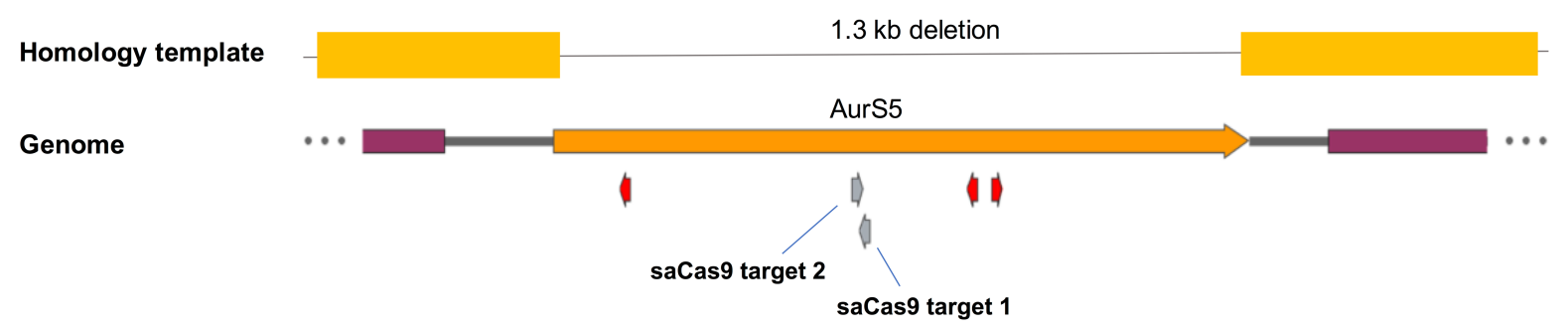

Figure 4. Scheme of targets and homology repair template used for S. roseosporus deletion.

Red and grey arrows indicate the locations of spCas9 and saCas9 targets tested respectively. 


\section{Supplementary data - Characterization of Cas proteins for CRISPR-Cas editing in streptomycetes}

Wan Lin Yeo ${ }^{1 \#}$, Elena Heng ${ }^{2 \#}$, Lee Ling Tan ${ }^{2}$, Yi Wee Lim, ${ }^{3}$ Yee Hwee Lim ${ }^{3}$, Shawn Hoon ${ }^{2}$, Huimin Zhao $^{4}$, Mingzi M. Zhang ${ }^{1,5^{*}}$, Fong Tian Wong ${ }^{2^{*}}$

1. Metabolic Engineering Research Laboratory, Institute of Chemical and Engineering Sciences, $A^{*}$ STAR, Singapore

2. Molecular Engineering Laboratory, Biomedical Institutes of Sciences, $A^{*} S T A R$, Singapore

3. Organic Chemistry, Institute of Chemical and Engineering Sciences, $A^{*} S T A R$, Singapore

4. Department of Chemical and Biomolecular engineering, Department of Chemistry, Department of

Biochemistry, University of Illinois at Urbana-Champaign, United States

5. Institute of Molecular and Genomic Medicine, National Health Research Institutes, Taiwan, R.O.C.

${ }^{\#}$ Contributed equally

*Correspondence should be directed to: zhangmz@nhri.org.tw, ftwong@bmsi.a-star.edu.sg

Supplementary Table 1. Oligonucleotides used in this study.

Figure S1. Representative trace of edited genome sequence (insertion of kas $O^{*} \mathrm{p}$ ) for Streptomyces sp. NRRL-244.

Figure S2. Representative trace of edited genome sequence (deletion of aurS5) for Streptomyces roseosporus 
Supplementary Table 1. Oligonucleotides used in this study.

\begin{tabular}{|c|c|}
\hline Sequence & Comments \\
\hline \multicolumn{2}{|c|}{ Diagnostic PCR and sequencing } \\
\hline ggcctcgaactccagcacctcgacg & \multirow{2}{*}{$\begin{array}{c}\text { PCR of target genomic locus for } S \text {. albus indigoidine } \\
\text { cluster }\end{array}$} \\
\hline cacgcgttcatggtgcccggcatc & \\
\hline cgcagcgtcgcggtcacctacgg & $\begin{array}{l}\text { Sequencing primers for edited genomic region ( } S \text {. } \\
\text { albus indigoidine cluster) }\end{array}$ \\
\hline tggcgtacttgtgctcctcggtccagagct & \multirow{2}{*}{$\begin{array}{c}\text { PCR of target genomic locus for S. sp. NRRL S-244 } \\
\text { cluster }\end{array}$} \\
\hline ACACGCTGTGGGTGTTCCTCCCGTACGT & \\
\hline GACATCGATCAGGAATCCGTGGTGCAGG & $\begin{array}{l}\text { Sequencing primers for edited genomic region (S. sp. } \\
\text { NRRLS-244 cluster) }\end{array}$ \\
\hline tggaccatcctcagctggaagaacct & \multirow{2}{*}{$\begin{array}{c}\text { PCR of target genomic loci for S. roseosporus aurS5 } \\
\text { deletion }\end{array}$} \\
\hline agatgatgggctgctcccagacccgta & \\
\hline gtgatcggaaatagtctgctgccgat & $\begin{array}{c}\text { Sequencing primer for edited genomic region ( } S . \\
\text { roseosporus aur } 55 \text { deletion) }\end{array}$ \\
\hline \multicolumn{2}{|c|}{ Construction of editing plasmids } \\
\hline ACGCgcagggttaccggacggcccg & \multirow{2}{*}{$\begin{array}{l}\text { PS sequence for Sth1Cas9 (S. albus indigoidine } \\
\text { cluster) }\end{array}$} \\
\hline AAACcgggccgtccggtaaccctgc & \\
\hline $\begin{array}{l}\text { TCTAGAGCTAGCATGCATATGAAGCTTATGAGGC } \\
\text { AGCGTGCCGGATTCGACTC }\end{array}$ & \multirow{2}{*}{$\begin{array}{l}\text { PCR of flank for Sth1Cas9 \& FnCpf1 (S. albus } \\
\text { indigoidine cluster) }\end{array}$} \\
\hline $\begin{array}{l}\text { AAAGCAGAGACCGTTCGAATGTGAACACCGGAC } \\
\text { GGCCCGGGGGAAGAG }\end{array}$ & \\
\hline $\begin{array}{l}\text { AACTACACGACTGGATACTGACTTTTCACCGCTT } \\
\text { CCCCAGGAAGTGGATCTTCAG }\end{array}$ & \multirow{2}{*}{$\begin{array}{l}\text { PCR of right flank for Sth1Cas9 \& FnCpf1 (S. albus } \\
\text { indigoidine cluster) }\end{array}$} \\
\hline $\begin{array}{l}\text { aatacgacagcgtgcaggactgggggagttATGagcaccagcacg } \\
\text { ccgccacccg }\end{array}$ & \\
\hline $\begin{array}{l}\text { acgcGTCTAAGAACTTTAAATAATTTCTACTGTTGT } \\
\text { AGATccggacggcccgggggaagagctg }\end{array}$ & \multirow{2}{*}{ PS sequence for FnCpf1 (S. albus indigoidine cluster) } \\
\hline $\begin{array}{l}\text { GAGTcagctcttcccccgggccgtccggATCTACAACAGTA } \\
\text { GAAATTATTTAAAGTTCTTAGAC }\end{array}$ & \\
\hline ACGCccatcgcagatcacacactcg & \multirow{4}{*}{ PS sequence for SaCas9 (S. albus indigoidine cluster) } \\
\hline AAACcgagtgtgtgatctgcgatgg & \\
\hline $\begin{array}{l}\text { GAGTtcaacggaactgaacgacgcaaacATCTACAACAGT } \\
\text { AGAAATTATTTAAAGTTCTTAGAC }\end{array}$ & \\
\hline AAACacaccaccgtcaatgtgttga & \\
\hline ACGCTCCCGTGGGTGAGACATTAC & \multirow{2}{*}{ PS sequence for spCas9 ( $S$. sp. NRRL S-244 cluster) } \\
\hline AAACGTAATGTCTCACCCACGGGA & \\
\hline $\begin{array}{l}\text { ACACGACTGGATACTGACTTTTCACACTAGtcggcc } \\
\text { agctccggagtggaatagc }\end{array}$ & \multirow{2}{*}{ PCR of left flank (S. sp. NRRL S-244 cluster) } \\
\hline $\begin{array}{l}\text { atacgacagcgtgcaggactgggggagttATGCTGGACGATC } \\
\text { TCCAGTTCGATGTATTCG }\end{array}$ & \\
\hline $\begin{array}{l}\text { gtcaaagcagagaccgttcgaatgtgaacaACGCAGGGGTG } \\
\text { GTGACCCCTGAGGC }\end{array}$ & \multirow{2}{*}{ PCR of right flank (S. sp. NRRL S-244 cluster) } \\
\hline $\begin{array}{l}\text { TCTAGAgctagcatgcatatgaagcttATCTGCACGACCTC } \\
\text { GACTGGGAGGCCGAG }\end{array}$ & \\
\hline $\begin{array}{l}\text { acgcGTCTAAGAACTTTAAATAATTTCTACTGTTGT } \\
\text { AGATCGGGGGCCATCACATGCTGGACGA }\end{array}$ & \multirow{2}{*}{ PS sequence for FnCpf1 (S. sp. NRRL S-244 cluster) } \\
\hline $\begin{array}{l}\text { GAGTTCGTCCAGCATGTGATGGCCCCCGATCTA } \\
\text { CAACAGTAGAAATTATTTAAAGTTCTTAGAC }\end{array}$ & \\
\hline ACGCCTCACCCACGGGAAAATACGC & \multirow{3}{*}{ PS sequence for SaCas9 (S. sp. NRRL S-244 cluster) } \\
\hline AAACGCGTATTTTCCCGTGGGTGAG & \\
\hline AAACGAGCTGACCGCGTCCGCCGCC & \\
\hline AAACtctccgaactcctcgccgga & \\
\hline
\end{tabular}


bioRxiv preprint doi: https:/doi.org/10 1101/526996: this version posted January 22 2019. The copyright holder for this preprint (which was not certified by peer review) is the author/funder, who has granted bioRxiv a license to display the preprint in perpetuity. It is made available under aCC-BY-NC-ND 4.0 International license.

\begin{tabular}{|c|c|}
\hline ACGCagcatctccttcgccatgga & $\begin{array}{c}\text { PS1 sequence for SpCas9 (S. roseosporus aurS5 } \\
\text { deletion) }\end{array}$ \\
\hline ACGCATCGATCCGTTTCTGCAGAC & \multirow{2}{*}{$\begin{array}{c}\text { PS2 sequence for SpCas9 (S. roseosporus aurS5 } \\
\text { deletion) }\end{array}$} \\
\hline AAACGTCTGCAGAAACGGATCGAT & \\
\hline ACGCGCTCGGCGTGTCCAACCGTC & \multirow{2}{*}{$\begin{array}{c}\text { PS3 sequence for SpCas9 (S. roseosporus aurS5 } \\
\text { deletion) }\end{array}$} \\
\hline AAACGACGGTTGGACACGCCGAGC & \\
\hline ACGCtgaccgagtggetcggccggc & \multirow{2}{*}{$\begin{array}{c}\text { PS2 sequence for SaCas9 (S. roseosporus aurS5 } \\
\text { deletion) }\end{array}$} \\
\hline AAACgccggccgagccactcggtca & \\
\hline ACGCactcggtcatccactcggcga & \multirow{2}{*}{$\begin{array}{c}\text { PS2 sequence for SaCas9 (S. roseosporus aurS5 } \\
\text { deletion) }\end{array}$} \\
\hline AAACtcgccgagtggatgaccgagt & \\
\hline $\begin{array}{l}\text { TCTAGAGCTAGCATGCATATGAAGCTTtgcgcgcgtc } \\
\text { gtcgttgacgtgc }\end{array}$ & \multirow{2}{*}{ PCR of left flank (S. roseosporus aurS5 deletion) } \\
\hline $\begin{array}{l}\text { acacgactggatactgacttttcacACTAGTgcgcatgcagttctcc } \\
\text { aatagttcggc }\end{array}$ & \\
\hline $\begin{array}{l}\text { aactattggagaactgcatgcgcACTAGTcgctccgccgcgaccat } \\
\text { ttaggggtt }\end{array}$ & \multirow{2}{*}{ PCR of right flank (S. roseosporus aurS5 deletion) } \\
\hline $\begin{array}{l}\text { AACTACACGACTGGATACTGACTTTTCACcggatctt } \\
\text { cccggagtccaccgagta }\end{array}$ & \\
\hline
\end{tabular}

BstBI

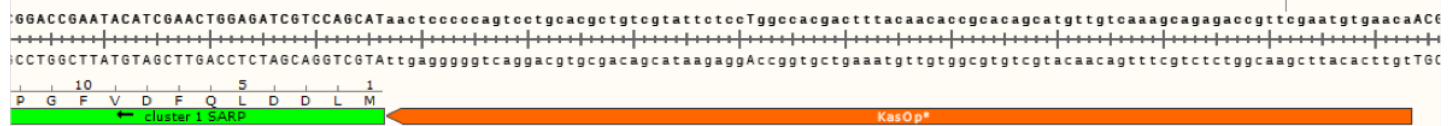

:GGACCGAATACATCGAACTGGAGATCGTCCAGCATaactococoagtectgoacgotgtegtattotoctggecacgactt tacaacacogcacagcatgttgtoaageagagacogttegaatgtgaacaACE :GGACCGAATACATCGAACTGGAGATCGTCCAGCATAACTCCCCCAGTCCTGCACGCTGTCGTATTCTCCTGGCCACGACTTTACAACACCGCACAGCATGTTGTCAAAGCAGAGACCGTTCGAATGTGAACAACG

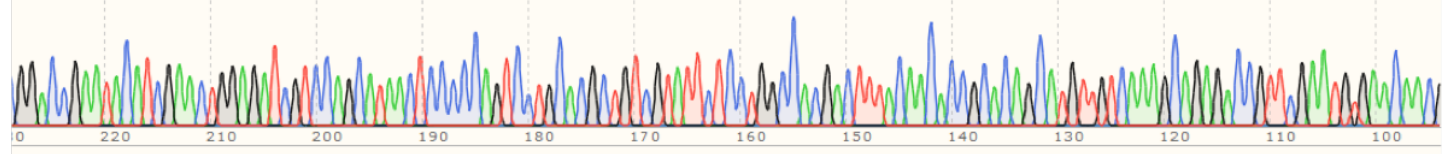

Figure S1. Representative trace of edited genome sequence (insertion of kasO* $\mathrm{p}$ ) for Streptomyces sp. NRRL S-244.

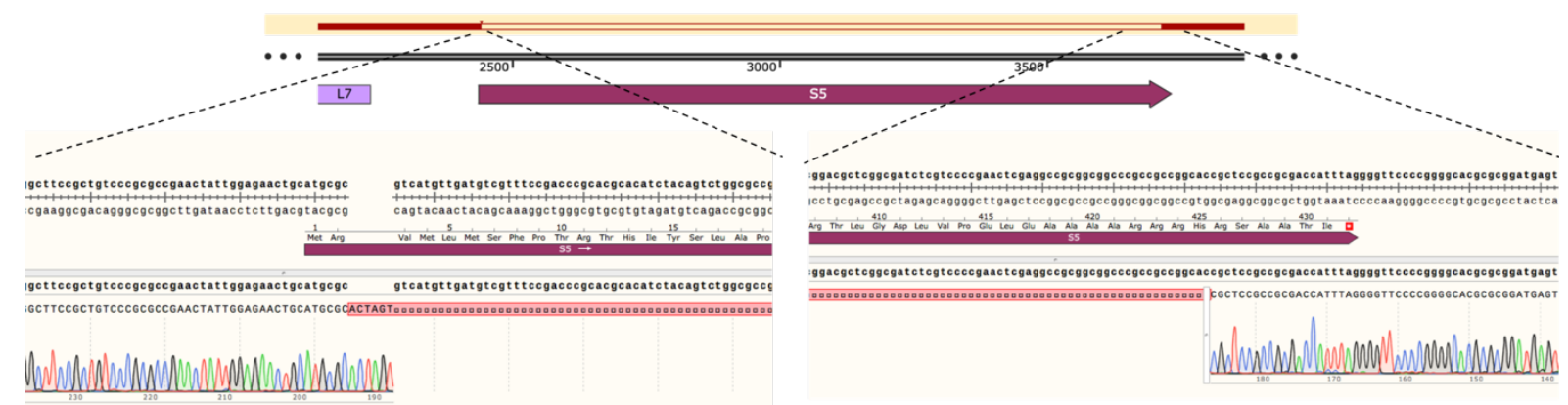

Figure S2. Representative trace of edited genome sequence (deletion of aurS5) for Streptomyces roseosporus. 